\title{
TRANSHUMANISMO E INTELIGENCIA ARTIFICIAL: EL PROBLEMA DE UN LÍMITE ONTOLÓGICO
}

\author{
Leopoldo Tillería Aqueveque ${ }^{1}$ \\ Universidad Tecnológica de Chile (INACAP) \\ Universidad Bernardo O'Higgins (UBO) \\ https://orcid.org/0000-0001-5630-7552 \\ E-mail: leopoldo.tilleria@inacapmail.cl
}

\section{RESUMEN:}

Se discute el problema del límite ontológico de la Inteligencia Artificial y del transhumanismo en contrastación con la ontología del Homo sapiens. Más allá de la llamada integración exógena o endógena (con sus prototipos respectivos, el androide y el ciborg), el escenario de una singularidad tecnológica parece materializarse en entidades que sintetizan biología y tecnología, por ejemplo, mediante una descarga o transbiomorfosis que traduzca las redes neuronales de nuestra mente a la memoria de un ordenador. Se trata de una hibridez que nos avisa sobre el advenimiento de nuevas especies que podrían dejar atrás al Homo sapiens. Si la síntesis ser humano/máquina es el límite deseado por el programa transhumanista, dicho límite parece haber cruzado, a su vez, el propio límite ontológico de lo humano, sobre el que hasta ahora se habían puesto más o menos de acuerdo -cada una presentando sus propias cartas- la ciencia, la filosofía y la religión.

PALABRAS ClaVE: Homo sapiens; Inteligencia Artificial; Ontología; Tecnología; Transhumanismo.

\section{TRANSHUMANISM AND ARTIFICIAL INTELLIGENCE: THE PROBLEM OF AN ONTOLOGICAL LIMIT}

\begin{abstract}
:
The problem of the ontological limit of Artificial Intelligence and transhumanism in contrast with the ontology of Homo sapiens is discussed. Beyond the so-called exogenous or endogenous integration (with their respective prototypes, the android and the cyborg), the scenario of a technological singularity seems to materialize in entities that synthesize biology and technology, for example, by means of a download or transbiomorphosis that translates the neural networks of our mind into the memory of a computer. This is a hybridization that warns us about the advent of new species that could leave Homo sapiens behind. If the human/machine synthesis is the limit desired by the transhumanist program, this limit seems to have crossed, in turn, the very ontological limit of the human, on which science, philosophy and religion had so far more or less agreed-each presenting their own cards.
\end{abstract}

KEYWORDS: Homo sapiens; Artificial Intelligence; Ontology; Technology; Transhumanism.

\footnotetext{
1 Doctor(a) en Filosofía por la Universidad de Chile (UCHILE) - Santiago - Chile. Académico e investigador del Área de Tecnologías de la Información y Ciberseguridad de Universidad Tecnológica de Chile (INACAP), Temuco, Chile; Académico e investigador de la Universidad Bernardo O'Higgins (UBO), Santiago, Chile.
}

AQUEVEQUE, Leopoldo Tillería. Transhumanismo e Inteligencia Artificial: el problema de un límite ontológico. Griot : Revista de Filosofia, Amargosa - BA, v.22 n.1, p.59-67, fevereiro, 2022. 


\title{
Introducción
}

En su ensayo Reglas para el parque humano (1999), el filósofo alemán Peter Sloterdijk realiza una crítica frontal al segundo Heidegger a propósito de su célebre Carta sobre el humanismo (2000), en la que este último se interrogaba si la catástrofe del mundo actual (plena posguerra) no se debería en realidad a la maniobra del ser humano de construir sus propios sistemas de autoelucidación y autoensalzamiento metafísico. En un pasaje decisivo de su ensayo, el filósofo de Karlsruhe hace la siguiente observación, que bien podría tenerse como una suerte de "manifiesto transhumanista":

\begin{abstract}
Por su efecto retrospectivo, un código tal [referido a las antropotécnicas] cambiaría también el significado del humanismo clásico, pues con él se publicaría y registraría que la humanitas no sólo implica la amistad del hombre con el hombre, sino también -y de modo crecientemente explícito- que el ser humano representa el más alto poder para el ser humano (SLOTERDIJK, 1999, p. 16).
\end{abstract}

Dentro de las numerosas definiciones de transhumanismo que la literatura reciente puede ofrecer, consideraré en este trabajo, en primer lugar, la definición que sistematiza el filósofo chileno Raúl Villarroel (2015), esto es: “[...] la posición de quienes creen que es posible provocar deliberadamente un 'mejoramiento' (enhancement) de los seres humanos, con miras a alcanzar un estado superior, a veces llamado 'transhumano', o incluso "posthumano" (p. 179). Probablemente, lo novedoso de esta aproximación no sea el concepto de "mejoramiento", puesto que la idea de mejora o terapia, o superación, ha estado presente en la mente del hombre desde el inicio de los tiempos, sino, en mi opinión, la noción de "estado superior".

Si nos fijamos bien, entre las observaciones de Sloterdijk y de Villarroel existe un punto en común: la condición de estado de poder o de superioridad que el ser humano pudiera o, para decirlo más problemáticamente, "debiera" alcanzar en su deriva como especie. Dicho horizonte de mejora podría entenderse, entonces, como algo parecido a un mandato "técnico" que al hombre le sería propio por naturaleza.

Quisiera abordar la pregunta por los límites de estas estrategias inmunológicas, dado que, si seguimos a Sloterdijk, de lo que se trata es de que el hombre se blinde o acorace técnicamente respecto de los embates del ambiente, incluido en este él mismo como especie. Y uno de aquellos límites es precisamente el de la tecnología. En otras palabras, nos moveríamos dentro de la pregunta por las posibilidades tecnológicas que el ser humano puede o debe darse o está autorizado a proveerse bajo el imperativo de una mejora de su condición humana.

De manera que la segunda idea que forma nuestra suposición -la de Inteligencia Artificial (IA)- y sobre la que ya avisa el título del escrito, debería comprenderse formando parte del continuum antropotécnico que Sloterdijk y otros autores (Lewis Mumford, por ejemplo) reconocen como lo distintivo del Homo sapiens.

Si en sentido "débil" la IA puede definirse como la búsqueda del mejor programa agente en una arquitectura dada, las proyecciones técnico/tecnológicas del transhumanismo parecieran acoplarse sin mayores interferencias en la corriente filogenética de la humanidad. Sin embargo, si la versión de la IA es una versión "fuerte", es decir, se plantea no sólo la pregunta por el mejor agente disponible, sino que sobre todo demanda y activa una comparación ontológica y epistémica entre la arquitectura humana y la de la máquina, las implicancias de esta artificialidad supondrían necesariamente un proceso de germinación tecnológica en la sociedad de seguro insospechado.

Primeramente, amplificaré la definición de IA, de modo de disponer de un marco conceptual un poco más robusto y al mismo tiempo más flexible. Enseguida, discutiré los alcances

AQUEVEQUE, Leopoldo Tillería. Transhumanismo e Inteligencia Artificial: el problema de un límite ontológico. Griot : Revista de Filosofia, Amargosa - BA, v.22 n.1, p.59-67, fevereiro, 2022. 
del concepto de transhumanismo y las posibilidades que este supondría para futuros estados de la IA y viceversa. Este es el núcleo del "problema ontológico" que representaría la conexión transhumanismo/IA. Sobre el final del trabajo se confrontarán los conceptos de límite y de síntesis a propósito de la idea transhumanista de convertibilidad ontológica.

\section{Aproximación al concepto de Inteligencia Artificial}

Una primera entrada a la idea de IA reproduce las nociones "débil" / "fuerte" recién anticipadas. En efecto, y a modo de desglose de este esquema, Meseguer y López de Mántaras (2017) indican que la IA "débil" es la ciencia e ingeniería que permite diseñar y programar ordenadores de forma que realicen tareas que requieren inteligencia; en tanto que la IA "fuerte" sería la ciencia e ingeniería que posibilita replicar la inteligencia humana mediante máquinas. De manera que, según esta versión, la transición de una IA "débil" a otra "fuerte" dependería del diseño de la máquina inteligente, en cuanto a que su forma de inteligencia debiera ser esencialmente humana. Conviene aclarar que, según esta hipótesis, la inteligencia que se espera pudiera especificar una máquina, ya sea en su fase "débil" o en su fase "fuerte", "[...] debería tratarse de inteligencia de tipo general y no especializada, ya que la inteligencia de los seres humanos es de tipo general" (MESEGUER y LÓPEZ DE MÁNTARAS, 2017, p. 10).

También se ha entendido la IA como "[...] una disciplina académica relacionada con la teoría de la computación cuyo objetivo es emular algunas de las facultades intelectuales humanas en sistemas artificiales" (BENÍTEZ, ESCUDERO, KANAAN y MASIP RODÓ, 2014, p. 12). A partir de esta definición nos damos cuenta de que su delimitación permite englobar en su significado variables sensoriales y abstractas. Por un lado, estaría referida típicamente a procesos de percepción sensorial y a sus consiguientes procesos de reconocimiento de patrones, pero, por otro, a la resolución de problemas de carácter más abstracto como la demostración de teoremas matemáticos, la adquisición de lenguaje, el jugar ajedrez o la traducción automática de textos (BENÍTEZ, ESCUDERO, KANAAN y MASIP RODÓ, 2014, p. 12-13). Siguiendo este argumento, podemos deducir dos derivadas: 1) que la IA es una rama de la informática teórica, y 2) que un sistema de inteligencia artificial requiere de una secuencia finita de instrucciones que especifique las diferentes acciones que ejecuta un ordenador para resolver un determinado problema, secuencia denominada estructura algorítmica del sistema de inteligencia artificial.

Ahora, desde una perspectiva más ontológica la IA puede ser comprendida como la ciencia que mediante el diseño de modelos computacionales tiene como objetivo último la construcción de un ente casi humano capaz de comunicarse en la misma lengua y con la misma competencia de sus interlocutores humanos (HERRERA y MUÑOZ, 2017, p. 157).

Por último, llama la atención la versatilidad del concepto que aporta Torra (2011), quien define la IA como una de las ramas de la Informática con fuertes raíces en otras áreas, como la lógica y las ciencias cognitivas. Lo cardinal de su acercamiento parece ser la conexión de la identidad de la IA con otras dimensiones cognitivas. Es decir, incorpora a la idea de una IA como parte de la Informática, otras cuatro condiciones que la dotan de una complejidad y una heurística que permiten reconocer enseguida ese «ente casi humano capaz de comunicarse en la misma lengua», descrito por Herrera y Muñoz (2017). En efecto, con base en el comportamiento humano la IA supondría:

i) Actuar como las personas (el modelo a seguir para la evaluación de los programas es el propio comportamiento humano).

ii) Razonar como las personas (se trata de desarrollar sistemas que razonen del mismo modo que las personas, tal como lo hace la ciencia cognitiva). 
iii) Razonar racionalmente (se parte de la premisa de que existe una forma racional de razonar: en este caso, la lógica y sus mecanismos de formalización del razonamiento).

iv) Actuar racionalmente (el objetivo son los resultados evaluados de forma objetiva; para ello es indiferente la forma de calcular el resultado, por ejemplo, en una partida de ajedrez) (TORRA, 2011, p. 2).

\section{La IA como problema ontológico}

La concepción de IA pergeñada en la sección anterior, en cualquiera de sus variantes, pone el acento en el problema del diseño de la máquina, al que podríamos llamar problema de la ontología de la IA. Es un problema, porque hasta ahora no ha quedado claro cuáles son aquellos atributos humanos que quedarían hipotéticamente fuera de las posibilidades heurísticas de una máquina inteligente. No hablemos de las capacidades de abstracción o creación, que han sido descritas con lujo de detalles por filósofos de la tecnología como Douglas Hofstadter, y que presentan las tareas de la creatividad, o, como también suele decirse, de la programación de la programación, fundamentadas en el concepto de analogía; es decir, donde sería necesaria la presencia de un repertorio preexistente para que la creatividad pueda manifestarse precisamente al diferenciarse de lo ya existente (PUGLISI, 1999, p. 70-71).

Otro caso de creatividad de una IA es el que plantea la performance artística de la famosa robot Sophia. Hace poco la ginoide sorprendió al mundo con una publicitada subasta de su primera obra de arte (una pintura en material no fungible). Y aunque desde el punto de vista de la "creación artística" la obra se presenta en el marco de una co-creación con el artista digital italiano Andrea Bonaceto, lo que queda para la posteridad es que el "genio" artístico también puede provenir del lado "más allá" de lo humano. De este modo, lo que corrobora esta experiencia es que la capacidad creativa, el fundamento estético de una obra, cualquiera sea su formato o lenguaje, incluso el estilo en el que se plasme, puede ser estrictamente robótica, o, para decirlo en los términos de la posmodernidad, "inclusiva" con la IA.

Ahora bien, esta interpretación de la IA como una entidad orientada al cálculorazonamiento-acción, parece demostrar que la misma pregunta por la naturaleza de la IA queda hoy en entredicho o, al menos, asociada a una inesperada equivalencia ontológica con la naturaleza del ser humano. Atendamos, por ejemplo, el inquietante concepto de persona sugerido por el médico y filósofo estadounidense H. Tristram Engelhardt (1995):

Las personas, no los seres humanos, son especiales -al menos si sólo se dispone de una moralidad secular general. Los seres humanos adultos competentes tienen una categoría intrínseca moral mucho más elevada que los fetos humanos o que incluso los niños pequeños. Es importante conocer la naturaleza de estas desigualdades con cierto detalle $[\ldots]$ (p. 151).

Como vemos, la estructura de diferencias entre los seres humanos (modelo que la posmodernidad ha hecho aún más patente mediante un discurso que pontifica acerca del valor de la diversidad de las "especies") parece re-definir el concepto de persona, asumiendo como nuevo núcleo ontológico la noción de "diferencia". Una consecuencia natural y hasta esperable de esta bioética es la consideración de nuevas entidades que puedan ser concebidas como personas. Es el caso, en principio, de la robótica social. En efecto, la posibilidad de formar parte del conglomerado de entidades susceptibles de ser rotuladas como personas alcanzaría a toda aquella gama de bots que cumplan con la "conjetura Engelhardt". Tal requisito está centrado en la capacidad autorreflexiva y en el ejercicio de una determinada racionalidad respecto del entorno. Sostiene el estadounidense: 
No todos los seres humanos son personas, no todos son autorreflexivos, racionales o capaces de formarse un concepto de la posibilidad de culpar o alabar. Los fetos, las criaturas, los retrasados mentales profundos y los que se encuentran en coma profundo son ejemplos de seres humanos que no son personas (ENGELHARDT, 1995, p. 155).

Pues bien, y en relación a un argumento que conecte la robótica social y el concepto de persona postulado por Engelhardt, es posible suponer que Sophia encajaría cómodamente dentro de una ética secular como la que demanda el filósofo, dándole, además, cabida práctica a una ontología inmanente y políticamente neutral como la suya. En otras palabras, Sophia sería una entidad que calificaría como persona bajo la etiqueta de "extraños morales", que Engelhardt postula paradójicamente como imperativo para una ética posmoderna.

De lo que se trata, pues, con esta "sustancialización extrema" de una ética planetaria, es de satisfacer la necesidad de que exista una sociedad pluralista, secular y pacífica donde los "extraños morales" o, en este caso, "extraños ontológicos", tengan cabida y puedan encontrarse sin mayor perturbación. Por eso, el filósofo americano puede decir que "[...] lo que, en términos seculares generales, es importante acerca de nosotros mismos como seres humanos, es el hecho de que seamos personas, no nuestra pertenencia a la especie Homo sapiens como tal" (ENGELHARDT, 1995, p. 156).

Pero la ontología de la IA no sólo muestra un cariz problemático en virtud de la disputa bioético-tecnológica entre los, por así decir, partidarios de la emancipación política de Sophia y quienes proclaman -sobre todo desde la vereda creacionista- algo así como la inviolabilidad de la naturaleza humana. La cuestión avanza en el propio terreno de la neurobiología. Dado que las ciencias cognitivas están todavía a mitad de camino de una completa comprensión de los extremadamente complejos procesos bioquímicos que ejecuta el cerebro humano, el desarrollo de la IA tendrá que esperar hasta fines de siglo para establecer una convergencia más significativa entre los procesos de una mente humana y los de una mente artificial:

\begin{abstract}
As such, there is not much that designers of algorithms can emulate from, especially given that machine learning still operates exclusively from the realm of statistics; that too on silicon-based computer systems, which are radically dif ferent from biological brains. A more meaningful convergence between the fields of AI and neuroscience is expected to unfold later this century, as we break into the "black box" and seek to understand the human brain in greater depth [Por lo tanto, los diseñadores de algoritmos no pueden emular mucho, sobre todo teniendo en cuenta que el aprendizaje automático sigue operando exclusivamente desde el ámbito de la estadística, y además en sistemas informáticos basados en el silicio, que son radicalmente diferentes de los cerebros biológicos. Se espera que a finales de este siglo se produzca una convergencia más significativa entre los campos de la IA y la neurociencia, a medida que vayamos entrando en la "caja negra" y tratemos de comprender el cerebro humano en mayor profundidad] (MIAILHE \& HODES, 2017, p. 8).
\end{abstract}

En realidad, se trataría (esta posibilidad de equivalencia heurística más definitiva entre la IA y el cerebro humano) de una imposibilidad ontológica, no sólo por la insuficiente evidencia científica sobre lo que pudiéramos llamar unas ciencias cognitivas con poderes predictivos sobre la relación entre los hechos y nuestros sistemas de creencias y actitudes, sino porque persiste entre los adherentes de la IA una suerte de complejo del Cyber sapiens. Bajo esta premisa, se esperaría que mientras mayor cantidad de información pudiera almacenar una máquina, más se asemejaría a una determinada heurística de los estados mentales del ser humano, independientemente del material físico que se utilice para configurar esta mente artificial. 
Como sea, la probabilidad de avanzar en la dimensión y diseño del Big Data muestra precisamente no una analogía, sino una configuración algorítmica diferenciada respecto del Homo sapiens:

El término clave aquí es el de "algoritmo". Cuanta más complejidad algorítmica, más capacidad predictiva, de ahí que todos los esfuerzos se centren en correlacionar infinidad de variables para acertar en las previsiones de futuro. El sueño inconfesable es lograr el "demonio de Laplace", esto es, una máquina que, previa recopilación de todas las variables posibles, sea capaz de predecir cualquier escenario de una forma completamente determinista (LÓPEZ, 2019, p. 10).

\section{Transhumanismo y convertibilidad ontológica}

Aunque en el transhumanismo coexisten distintas posiciones, lo decisivo es su creencia de que por medio de la ciencia y la tecnología los seres humanos podrían superar su situación actual e incluso dirigir voluntariamente la evolución de nuestra especie, teoría denominada Evolución Dirigida (VACCARI, 2014, p. 238). Posiblemente la definición más ajustada sea la de O'Connell (2017), quien entiende el transhumanismo como un movimiento de liberación de la naturaleza.

La idea que subyace a esta postura es la de que el Homo sapiens en su forma actual no solamente no representa el final de nuestra evolución, sino que, a decir verdad, es una fase muy temprana de la misma. De hecho, el proyecto transhumanista se llevará a cabo no sólo mediante ingeniería genética, eugenesia embrionaria y prenatal, nanotecnología y biotecnología aplicada al cerebro, sino también mediante la crioconservación de pacientes enfermos o fallecidos y la reanimación futura de pacientes en suspensión criogénica (POSTIGO, 2011). Incluso puede hablarse de un umbral transhumanista, donde prevalezca una suerte de transorganicidad técnica:

\footnotetext{
Superar las limitaciones biológicas de la naturaleza humana, su anclaje en y dependencia de la organicidad y el determinismo corporal es una de sus aspiraciones, junto con el logro de una superinteligencia, una superlongevidad y una superfelicidad que elevan a ideal ético de una humanidad que se respete a sí misma. Todo ello se resume en el término Mejoramiento (BOSTROM y SAVULESCU, 2017, cit. en SÁNCHEZ, 2021, p. 3).
}

Lo paradójico es que el transhumanismo, además de colocarse como centro de su propio análisis y experiencia, irónicamente vuelve a nociones teocéntricas en vez de antropocéntricas. Como lúcidamente advierte Sánchez (2021) a propósito de la idea de un segundo Edén: "Además de la identidad biónica y trans que aportan las biotecnologías, se anuncian y advienen las ciberidentidades [...]. La creación de robots, dicen, es el comienzo de un nuevo Edén, pues serán la última creación humana; las posteriores serán ya creación de los androides y de las máquinas" (p. 10).

El corolario de esta lógica viene a ser la tesis de que la muerte es una enfermedad curable, dando así un nuevo significado a la teoría de la trascendencia del ser humano. No olvidemos que desde esta misma esquina se ha promovido seriamente la posibilidad de que podamos integrarnos como especie con las tecnologías NBIC (Nanotecnología, Biotecnología, Tecnologías de la Información y la Comunicación, y Neurociencia) a modo de "mejoramiento definitivo". En cierto modo, el transhumanismo rescata aquellos deseos de trascendencia presentes en las narraciones míticas de antiguas culturas, y recurre al imaginario de ciencia ficción para explorar nuevas fronteras, precisamente a las tecnologías NBIC que la Cuarta Revolución Industrial provee, para volverlas una realidad palpable (GAYOZZO, 2021, p. 196).

Aquí me parece imprescindible citar a Koval (2011), en un comentario a medio camino entre la metafísica de la tecnología y la ciencia ficción: 
El Argumento del Fin del Mundo (Doomsday Argument), postulado así por autores como Bill Joy y Thomas Sturm respecto de las consecuencias que traerá, o podría traer, en un futuro cercano, el enorme desarrollo tecnológico, resulta precisamente de la llegada de una singularidad tecnológica expresada en la emergencia de los modernos autómatas antropomorfos inteligentes («robots universales» de Moravec, y «máquinas emocionales» de Kurzweil), entidades que nacen de la combinación explosiva del conjunto de Tecnologías de la Información y la Comunicación (que dan lugar a un aumento geométrico en la capacidad de cálculo y permiten una elaboración nanométrica de seres artificiales mitad máquina, mitad organismo) (p. 10).

No parece, pues, descabellado suponer que durante la segunda mitad de este siglo posiblemente vivamos el fin de la Edad Contemporánea y seamos testigos del inicio de una nueva, a la que perfectamente pudiésemos llamar, pensando en soluciones tecnológicas de última gama, Edad de Grafeno. Este paso de una era a otra estaría definido por avances aceleradísimos en materia de ciencia y tecnología (biología sintética, terapia genética, inteligencia artificial fuerte, nanotecnología, nanomedicina), que determinarían, a su vez, una reacción en cadena en varias otras disciplinas preocupadas del hombre y su entorno (sociología, psiquiatría, farmacología, bioética, ecología de las comunidades, etc.).

Dicho punto de inflexión está representado por la segura ratificación del estatus ontológico de entidades con un diseño que se ubicaría en todo el arco de la relación hombre/máquina. No es difícil suponer que nuestra evolución continuará justamente en esta nueva deriva ontológica, hasta llegar a una interfaz en que nuestros descendientes sean literalmente máquinas o clones con completa autonomía, superlinajes en los que el hombre querrá haber visto resueltas una serie de limitaciones biológicas, cognitivas e informacionales, y que lo habrán superado, por lejos, como especie más evolucionada del planeta. De hecho, hay dos fechas claves: la primera es 2029, donde los ordenadores adquirirán conciencia de sí mismos; la segunda es 2045, donde se producirá definitivamente el advenimiento de la singularidad, o, lo que es lo mismo, las máquinas serán más inteligentes que los humanos (SÁNCHEZ, 2015).

Es aquí cuando la pregunta ontológica se torna indispensable, no sólo por una cuestión, como quien dice, de hacer frente a un cierto especismo, sino porque la propia fórmula de una integración endógena o exógena, cuyos prototipos son el ciborg y el androide, respectivamente (KOVAL, 2011, p. 3-4), parece obsoleta o simplemente insuficiente respecto de ontologías emergentes fundadas en la noción de síntesis.

Una de estas ontologías, propiciada por el transhumanismo más radical, es aquella que pretende una abstracción absoluta de la materia orgánica, mediante una descarga o transbiomorfosis (metamorfosis transbiológica) que traduzca las redes neuronales de nuestra mente a la memoria de un ordenador (KOVAL, 2011, p. 13). Como observa Dery (1998):

Esta versión extrema del transhumanismo, conocida como poshumanismo trascendental, defiende la idea de un ser líquido-fluido posbiológico, abstracto, puro y sin anclajes al cuerpo, cuya supresión se hace necesaria; ser que reconoce en la sustancia limitaciones a su potencialidad, transferido tecnológicamente en la forma de conciencia a un sistema informático (cit. en KOVAL, 2011, p. 13).

Este es precisamente el ideario del filósofo transhumanista Nick Bostrom: consumar el abandono de la especie humana en pos de una nueva especie. Pero Bostrom (2002) va aún más lejos, planteándose la factibilidad de la tesis transhumanista con o sin argumento creacionista de por medio, es decir, considerando al transhumanismo como un proyecto viable aún si se partiese de la hipótesis de un diseño original: 
For people who are already convinced that there is a God, however, the design hypothesis is likely to appear as an attractive explanation of why our uni-verse is fine-tuned. And if one is not already convinced about the existence of a Designer, but thinks that it is a coherent possibility, one may be tempt-ed to regard fine-tuning as a reason for increasing one's credence in that hypothesis [Sin embargo, para las personas que ya están convencidas de que existe un Dios, es probable que la hipótesis del diseño aparezca como una explicación atractiva de por qué nuestro universo está ajustado. Y si uno no está ya convencido de la existencia de un Diseñador, pero piensa que es una posibilidad coherente, puede verse tentado a considerar el ajuste fino como una razón para aumentar la credibilidad de uno en esa hipótesis] (p. 12).

\section{Conclusiones}

El concepto de singularidad tecnológica, materializado en entidades que pueden sintetizar sin mayores problemas biología y tecnología, nos pone en aviso sobre el advenimiento de nuevas especies -probablemente híbridas- que pudieran dejar atrás todo rastro del Homo sapiens. Nada nuevo, si tenemos en cuenta que hace rato el transhumanismo persigue borrar la línea que separa a los humanos y los dispositivos tecnológicos bajo la hipótesis de que la mente puede sobrevivir sin un soporte como el cerebro. Por tanto, si la síntesis ser humano/máquina o biología/tecnología es el límite deseado por el programa transhumanista, dicho límite parece haber cruzado, paradójicamente, el propio límite ontológico de lo humano, sobre el que hasta ahora se habían puesto más o menos de acuerdo la ciencia, la filosofía y la religión.

La ciencia parece haber dado el primer paso en este camino de "convertibilidad ontológica", a partir del desarrollo de su hija predilecta: la tecnología. Sin embargo, la filosofía ya había advertido de esta misma posibilidad hace varios siglos, por ejemplo, cuando el marqués de Condorcet se preguntaba en 1795 si sería absurdo imaginar que la mejora de la especie humana debiera ser considerada como susceptible de progreso indefinido e ilimitado (CARDOZO y MENESES, 2014). En cualquier caso, y es en este terreno donde seguramente se dará la "madre de todas las batallas", queda aún por escuchar qué tiene qué decir la bioética como hija putativa de la filosofía.

Y, por último, queda la religión, el bastión de la fe. El eje creacionista, distribuido en el sinfín de ámbitos propios de la razón, el arte y la religión, de seguro abogue por la no transgresión de este límite y quiera resguardar el principio bíblico de una criatura hecha a semejanza del Creador. Curiosamente, para desbaratar este argumento trascendental no es necesario rechazar la religión, ni siquiera la idea de Dios. Basta, como se podrá deducir, con reordenar las piezas del tablero (el "ajuste fino" sugerido por Bostrom). Dicho eufemísticamente, ponerse en el lugar del Creador y diseñar el tipo de criatura (no sabemos si con o sin libre albedrío) que encaje en esta idea de universo posthumano. 


\section{Referencias}

BENÍTEZ, Raúl, ESCUDERO, Gerard, KANAAN, Samir y MASIP RODÓ, David. Inteligencia artificial avanzada. Barcelona: UOC, 2014.

BOSTROM, Nick. Anthropic Bias: Observation Selection Effects in Science and Philosophy. New York: Routledge, 2002.

CARDOZO, John \& MENESES, Tania. Transhumanismo: concepciones, alcances y tendencias. Análisis, vol. 46, n. 84, p. 63-88. 2014.

ENGELHARDT, Hugo Tristram. Los fundamentos de la bioética. Madrid: Paidos, 1995.

GAYOZZO, Piero. Singularidad tecnológica y transhumanismo. Teknokultura. Revista de Cultura Digital y Movimientos Sociales, vol. 18, n. 02, p. 195-200, 2021.

HEIDEGGER, Martin. Carta sobre el humanismo. Madrid: Alianza, 2000.

HERRERA, Leandro y MUÑOZ, Diego. Inteligencia Artificial y lenguaje natural. Lenguas Modernas, vol. 19, p. 157-165, 2017.

KOVAL, Santiago. Convergencias tecnológicas en la era de la integración hombre-máquina. Razón y Palabra, n. 75, p. 1-17, 2011.

LÓPEZ, Manuel. Las narrativas de la inteligencia artificial. Revista de Bioética y Derecho, n. 46, p. 5-28, 2019.

MESEGUER, Pedro y LÓPEZ DE MÁNTARAS, Ramón. Inteligencia Artificial. Madrid: CSIC, 2017.

MIAILHE, Nicolas \& HODES, Cyrus. The Third Age of Artificial Intelligence. Field Actions Science Reports, n. 17, p. 6-11, 2017.

O’CONNELL, Mark. To Be a Machine. Londres: Granta Books, 2017.

POSTIGO, Elena. Transhumanismo y Post-humano: principios teóricos e implicaciones bioéticas, 2011. Disponible en: <https://tinyurl.com/y7bzvzcw >. Acceso en: 10 de abril de 2021.

PUGLISI, Gianni. Un nuevo interlocutor en las relaciones entre filosofía y poesía: la estética computacional de Hofstadter. En: VATTIMO, Gianni (comp.). Filosofía y poesía: dos aproximaciones a la verdad. Barcelona: Gedisa, 1999, p. 65-74.

SÁNCHEZ, Teresa. La propuesta transhumanista para la abolición del género. Pieza del rediseño de la naturaleza humana. Aperturas Psicoanalíticas, n. 66, p. 1-22, 2021.

SÁNCHEZ, Carlos Manuel (09 de agosto de 2015). Transhumanismo: el último paso en la evolución humana. XL Semanal.

SLOTERDIJK, Peter. Reglas para el Parque Humano. Una respuesta a la "Carta sobre el Humanismo". FiloXarxa Diccionari enciclopèdic de filosofia, p. 1-21, 1999. Disponible en: < https://tinyurl.com/5fzaffju>. Acceso en: 04 de mayo de 2021.

TORRA, Vicenç. La inteligencia artificial. Lychnos. Cuadernos de la Fundación General CSIC, n. 07, p. 1-6, 2011.

VACCARI, Andrés. La posthumanidad como un bien objetivo: los peligros del futurismo en el debate sobre la optimización genética humana. Acta Bioethica, vol. 20, n. 02, p. 237-245, 2014.

VILLARROEL, Raúl. Consideraciones bioéticas y biopolíticas acerca del transhumanismo. El debate en torno a una posible experiencia posthumana. Revista de Filosofía, vol. 71, p. 177-190, 2015.

Autor(a) para correspondência / Corresponding author: Leopoldo Tillería Aqueveque. leopoldo.tilleria@inacapmail.cl

AQUEVEQUE, Leopoldo Tillería. Transhumanismo e Inteligencia Artificial: el problema de un límite ontológico. Griot : Revista de Filosofia, Amargosa - BA, v.22 n.1, p.59-67, fevereiro, 2022. 